\title{
Vibration propagation characteristics around defective part on concrete structure: Experiments on diagnosis based on phase delay in vibration response
}

\author{
Teruo Iwase ${ }^{1}$, Mareyasu Doi $^{1}$, Yutaka Mahide $^{2}$ and Tukasa $\mathrm{Abe}^{3}$ \\ ${ }^{1}$ Department of architecture, Niigata University, Niigata, 950-2181 Japan \\ e-mail:iwase@cc.Niigata-u.ac.jp \\ ${ }^{2}$ Nishihara Engineering Co. Ltd., Japan \\ ${ }^{3}$ Ministry of Land, Infrastructure and Transport, Japan
}

(Received 14 May 2001, Accepted for publication 11 June 2001)

Keywords: Non-destructive diagnosis, Crack, Exfoliation, Concreat structure, Laser vibrometer PACS number: 43.40.-r, 43.58.-e

\section{Introduction}

Authors examined the method observing vibration response by using laser vibrometer and made its effectiveness clear to detect and diagnose defective parts came out on a concrete structure. In other words, differences of the amplitude in vibration response around defective part as a crack or an exfoliation referring to that around sound part were extracted, and the possibility of realization of a nondestructive diagnosis method based on those results was examined. It had been mentioned that such a method would be very effective as the conclusion of those experiments already introduced [1-4].

In this paper, authors take attention of analysis to the phase delay data in vibration response instead of that we have taken to the only amplitude data in the previous studies. Then, strengths of the phase delay change in vibration responses around some defects are investigated, and it is examined whether a diagnosis based on the phase data can be applied to another non-destructive detecting method for defects.

\section{Outline of experiment for test concrete block}

Basic block diagram of the measurement is shown in Fig. 1. Some experiments were carried out by using three kinds of concrete block reinforced by fine steel bar, each has a single groove imitated a crack with each depth on its surface. Each dimension was common as $600(w) \times 300(d) \times 100(h)$ in unit of $\mathrm{mm}$, and each groove had the width of $1 \mathrm{~mm}$, the length of $300 \mathrm{~mm}$ and the depth of $0 \mathrm{~mm}, 20 \mathrm{~mm}$ and $40 \mathrm{~mm}$ for each. The test block without groove, i.e. the depth of $0 \mathrm{~mm}$, was prepared for reference. A miniature circular piezoelectric device was fixed by double-face adhesive tape on the surface of the test block nearby the groove, and random vibration was excited from it by using white noise. Twenty observation points were arranged in the interval of $5 \mathrm{~mm}$ and with the direction at right angles to the groove. Then, observation experiments of the vibration response for every point were carried out by using laser vibrometer and FFTanalyzer. The vibration responses are obtained by the transfer function analyses of observed vibration velocity signals against the original white noise signal. We think that our method using the laser vibrometer is very excellent as noncontact measurements, resonance free in sensor setting and as possibility at very high frequency. Finally, by the same way, field experiments were examined for the several cracks came out on a concrete wall of an actual building.

\section{Experiments on test concrete blocks}

3.1. Results of experiments

As for experimental results, the frequency characteristics of the phase delay at all observation points are presented by 3D type in Fig. 2, Fig. 3 and Fig. 4 when groove depth is $0 \mathrm{~mm}, 20 \mathrm{~mm}$ and $40 \mathrm{~mm}$ respectively. Observation results on these figures are as follow. In case of the groove depth of $0 \mathrm{~mm}$, gentle changes as the phase delay increasing in accordance with the propagation distance from the vibration source to the observation point are shown especially in the ultra sonic frequency range.

On the other hand, the difference of phase delay between at the 10-th observation point and the 11-th is remarkably large in high frequency range and in both cases of the test block with the groove depth of $20 \mathrm{~mm}$ and $40 \mathrm{~mm}$. Large phase delays can be still confirmed in the vibration responses at the observation points from 12-th to 15-th which are more distant than the 11-th point introduced just above. These characteristics are common in wide frequency range, and more remarkable at very high frequency as in the ultra sonic frequency range.

3.2. Detection of defects based on large change of phase delay

If it is taken attention to phase delay in the ultra sonic frequency range, some effective bases for the developing detection and diagnosis method of defect part can be found in the fact that large changes are clear. For examples, phase delay data in cases of grooves depth of $0 \mathrm{~mm}, 20 \mathrm{~mm}$ and $40 \mathrm{~mm}$ are compared in Fig. 5, and the difference between at the 10-th point and 11-th point is large as $2,000^{\circ}$ in case of the groove depth $20 \mathrm{~mm}$ and that is also large as $1,500^{\circ}$ in case of the groove depth $40 \mathrm{~mm}$. Such a remarkable discontinuous phase delay clearly corresponds to a defective part as crack on a concrete structure.

Therefore, authors think that these phase delay data are every useful to realization of non-destructive detection and diagnosis for defective parts, but we don't think that classification of groove depths is possible in the degree of depth as $40 \mathrm{~mm}$ or $20 \mathrm{~mm}$ by only the phase delay data. 


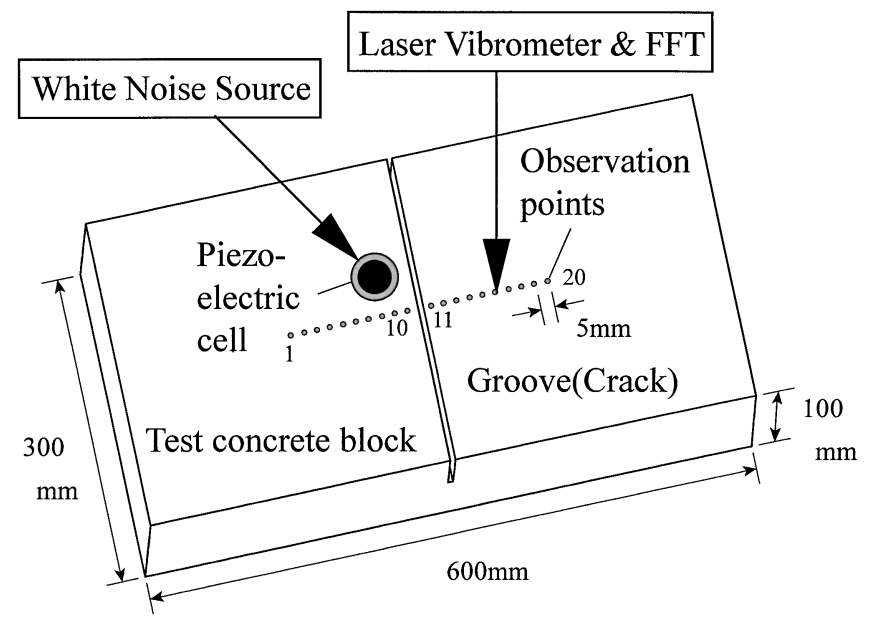

Fig. 1 Basic block diagram and arrangement of source point and observation points for experiments of vibration response on the test concrete block.

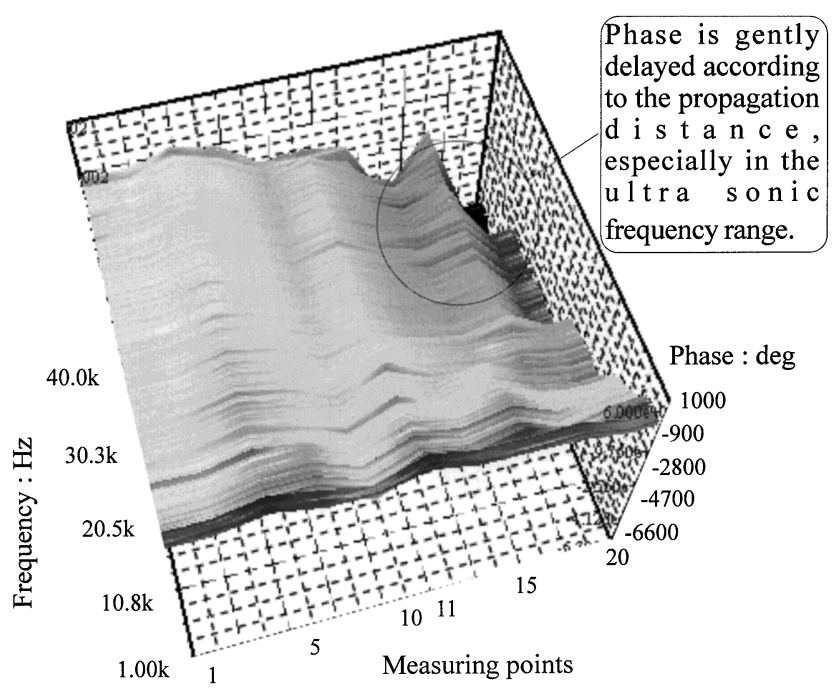

Fig. 2 The phase delays as functions of both frequency and observation point on the test concrete block without groove. Phase is gently delayed according to the propagation distance, especially in the ultra sonic frequency range.

\section{Application to detection on a concrete wall}

An experiment to confirm the actual validity of this technique was carried out. Its outline and results are as follow.

Twenty observation points were arranged in the interval of $5 \mathrm{~mm}$ and as keeping the right angle direction to a thin crack came out on a wall surface of a concrete building. Their arrangement with the vibration exciting points is shown in Fig. 6.

Analyzed frequency characteristics of phase delay for all observation point are shown by 3-D type display in Fig. 7. In this figure, remarkable phase changes between a pair of closed points in very wide frequency range can be seen as same as the results of model experiments shown in Fig. 3 and in Fig. 4 for imitating a crack. Then, averaged phase delay at the all observation points in the frequency range from 20 to $40 \mathrm{kHz}$

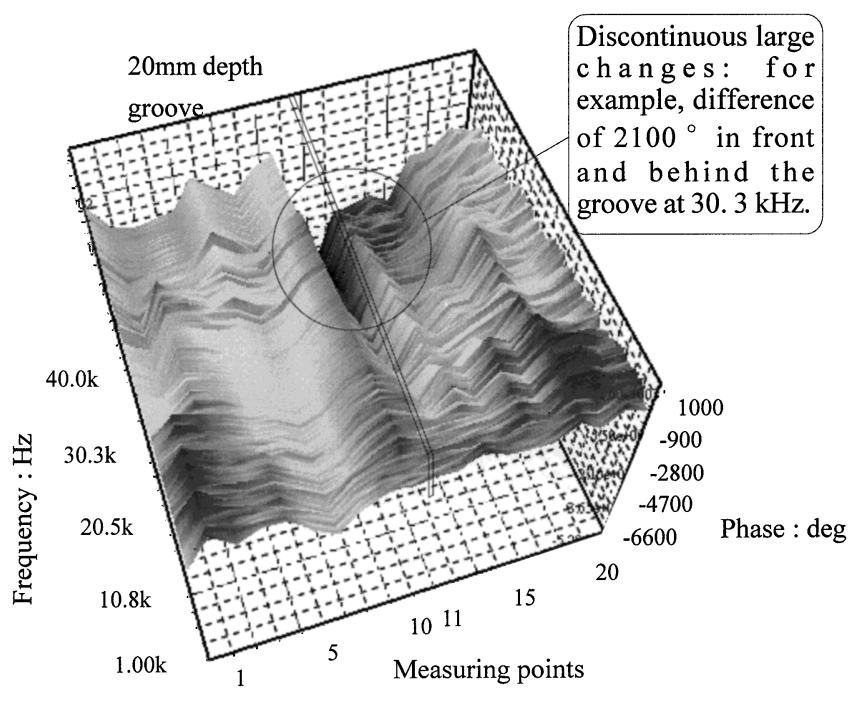

Fig. 3 The phase delays as functions of both frequency and observation point on the test concrete block with groove depth $20 \mathrm{~mm}$. Discontinuous large changes: for example, difference of $2,100^{\circ}$ in front and behind the groove at $30.3 \mathrm{kHz}$.

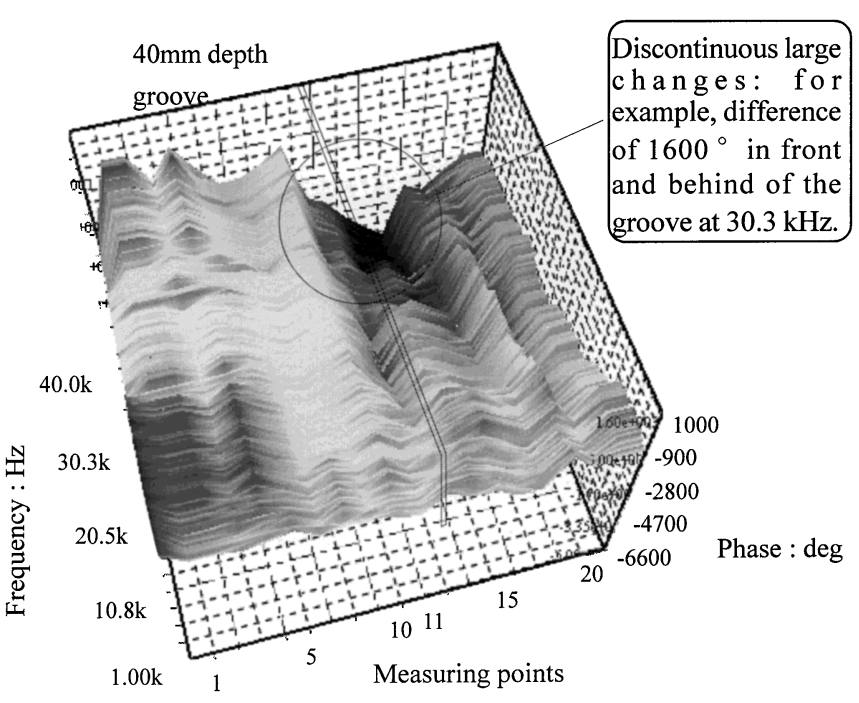

Fig. 4 The phase delays as functions of both frequency and observation point on the test concrete block with groove depth $40 \mathrm{~mm}$. Discontinuous large changes: for example, difference of $1,600^{\circ}$ in front and behind of the groove at $30.3 \mathrm{kHz}$.

is shown in Fig. 8, and difference between both observation point 10 and point 11 can be read as larger than $4,000^{\circ}$.

Such a correspondent relation between the position where a large change in phase delay can be observed and the position where a crack exists is the same relation as in the model experiments for imitating several kinds of crack.

\section{Conclusions}

From the experiments of the vibration response using several kinds of test concrete block with groove, imitated a crack, it confirmed that remarkable difference could be observed between both phase delay data at the points in front 


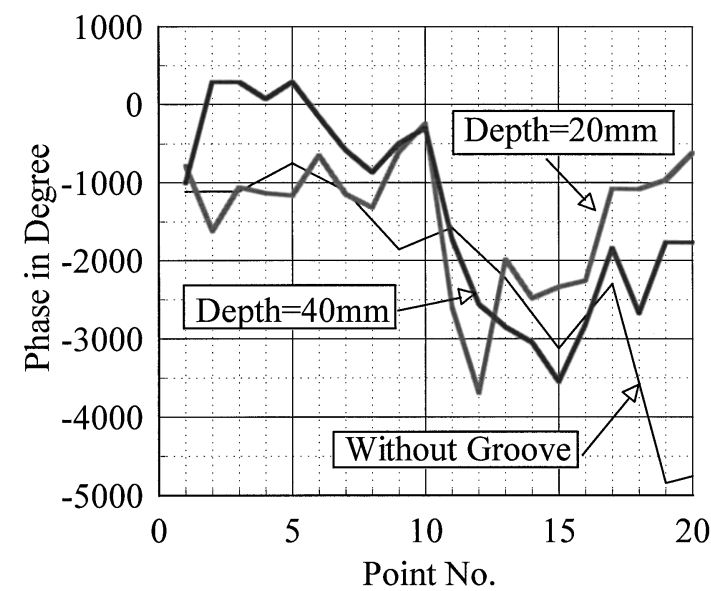

Fig. 5 The changes of phase delay at $30.3 \mathrm{kHz}$ around each groove on the test block.

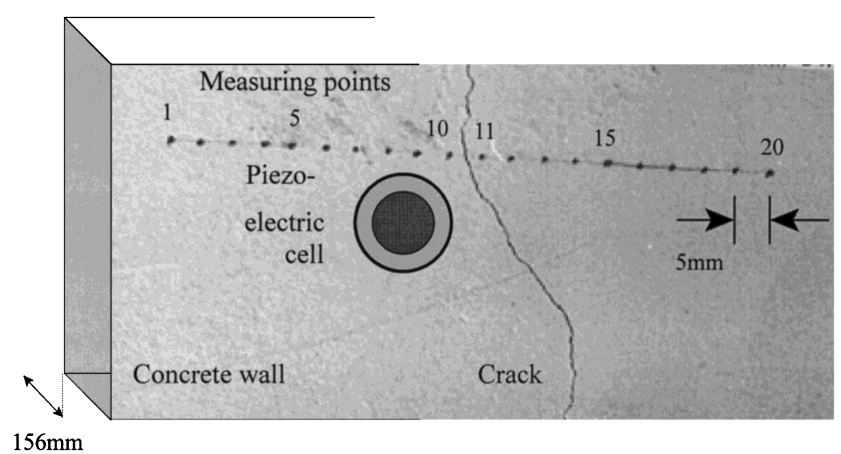

Fig. 6 Arrangement of source point and observation points for experiments of vibration response on an actual concrete wall.

and behind the groove especially in the ultra sonic frequency range. The clearly large change could be also recognized in phase delay data at the points around defective parts as crack in actual concrete wall.

It is the conclusion of this study that detection of a crack comes out on a wall of a concrete building is possible by observations of the vibration response with taking attention to the large changes of phase delay. Next target of this study is a developing the estimation method of the unknown crack depth by using other acoustical or vibrational techniques and by analysis at very higher frequencies more than those in this study by making best use of excellent points of laser vibrometer.

\section{References}

[1] T. Abe, Y. Mahide and T. Iwase, "Study on exciting method of ultrasonic vibration for non-destructive detection of crack in a building structure", Summ. Tech. Pap. Annu. Meet. Archit. Inst. Jpn., Vol. D-1, 361 (1999).

[2] Y. Mahide, T. Abe and T. Iwase, "Study on a new non-

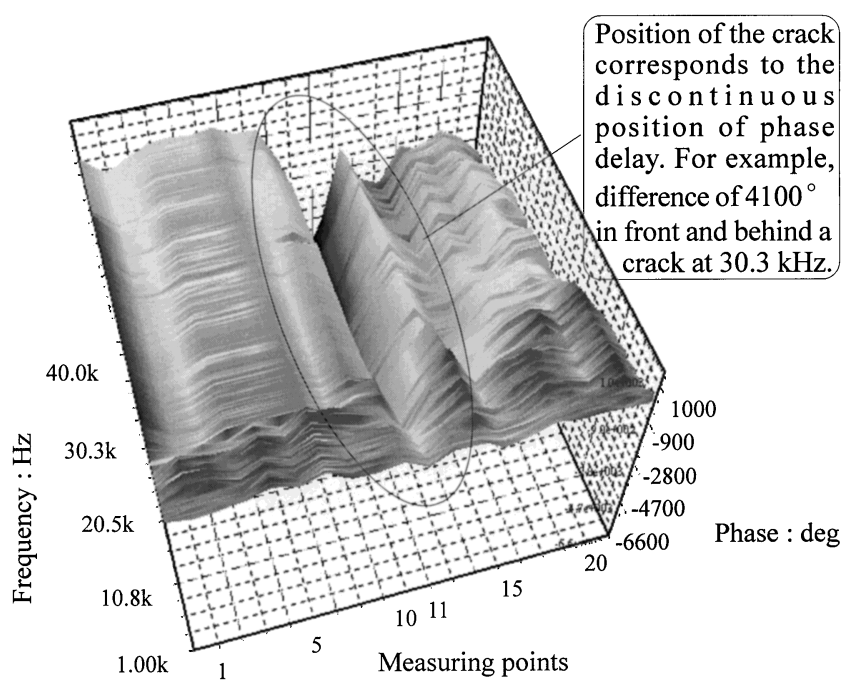

Fig. 7 Examples of phase delays as functions of both frequency and observation point around a crack on an actual concrete wall. Position of the crack corresponds to the discontinuous position of phase delay. For example, difference of $4,100^{\circ}$ in front and behind a crack at $30.3 \mathrm{kHz}$.

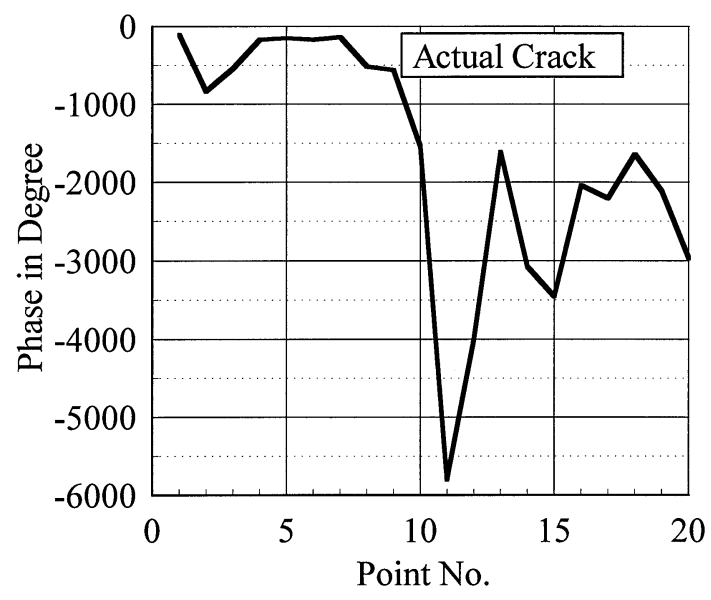

Fig. 8 The averaged phase delays in the range of 20 $40 \mathrm{kHz}$ at the points around a crack on an actual concrete structure.

destructive detection method by ultrasonic vibration", Summ. Tech. Pap. Annu. Meet. Archit. Inst. Jpn., Vol. D-1, 363 (1999).

[3] T. Iwase, Y. Mahide and T. Abe, "Study on a new nondestructive detection method of a crack and exfoliation on a concrete structure", Tech. Rep. Noise Vib. Acoust. Soc. Jpn., N2000-04, 1 (2000).

[4] T. Iwase, T. Abe and Y. Mahide, "Study on a non-destructive detection method of a crack and an exfoliation of a concrete structure by acoustic-vibration technique", Proc. Spring Meet. Acoust. Soc. Jpn., 121 (2000). 